\title{
ON RINGS IN WHICH EVERY IDEAL IS THE ANNIHILATOR OF AN ELEMENT
}

\author{
CLEON R. YOHE ${ }^{1}$
}

Let $R$ be a semisimple Artin ring (i.e. $R$ is a direct sum of matrix rings over division rings). Then if $L$ is a left ideal of $R, L=R e$ where $e$ is an idempotent and so $L$ is exactly the left annihilator of the element $1-e$. We investigate the structure of rings having this property. If $S$ is a subset of a ring $R$, let $e(S)$ and $r(S)$ denote the left and right annihilators of $S$. The notation $e_{R}(S)$ will be used when it is necessary to specify the ring $R$.

Definition. $R$ is a left elemental annihilator ring (l.e.a.r.) if, whenever $L$ is a left ideal of $R$, there exists an element $a \in R$ such that $L=e(a)$. A right elemental annihilator ring (r.e.a.r.) is defined analogously.

Notice that if $R$ is any ring (always assumed to have a unity element) and $S$ is a subset of $R$, then $e(S)=e(r(e(S)))$ and $r(S)$ $=r(e(r(S)))$. Hence if $R$ is a l.e.a.r., and $L$ is a left ideal, we have in particular that $L=e(r(L))$. If $R$ is a r.e.a.r., and $I$ a right ideal, then $I=r(e(I))$.

We consider first the commutative case. It is known [1, Theorem 1.1 ] that if $R$ is completely primary (local with nilpotent maximal ideal), then $R$ has the property that every ideal is the annihilator of some subset of $R$ if and only if $R$ has a unique minimal ideal. Using the methods of Theorem I below, it is possible to derive from this the fact that if $R$ is a commutative noetherian ring, then every ideal of $R$ is the annihilator of some subset if and only if $R$ is a direct sum of completely primary rings each of which has a unique minimal ideal. By imposing the more strenuous condition that $R$ actually be an elemental annihilator ring, we obtain a similar result without the hypothesis of the chain condition.

THEOREM I. Let $R$ be a commutative ring with unit element. Then $R$ is an elemental annihilator ring if and only if $R$ is a direct sum of completely primary principal ideal rings.

Proof. Let $R$ be a completely primary principal ideal ring, with maximal ideal $(m)$, where $m^{k}=0$. Any $r \in R$ has an expression $r=u m^{t}$, where $u$ is a unit of $R$ and $t$ is an integer, $0 \leqq t \leqq k$. It is clear

Received by the editors August 23, 1967.

1 This research was supported in part by National Science Foundation grant GP7175 . 
from this that the only ideals of $R$ are $(m),\left(m^{2}\right), \cdots,\left(m^{k}\right)=(0)$ and that for any $t,\left(m^{t}\right)=e\left(m^{k-t}\right)$. Thus $R$ is an elemental annihilator ring.

Let $R=S \oplus T$, where $S$ and $T$ are e.a.r.'s, and suppose that $I$ is an ideal of $R$, so $I=J \oplus L$, where $J$ is an ideal of $S$ and $L$ is an ideal of $T$. There exist $a \in S$ and $b \in T$ so that $J=e_{S}(a)$ and $L=e_{T}(b)$. Then $I=e_{S}(a) \oplus e_{T}(b)=e_{R}(a+b)$, which means that $R$ is an e.a.r. This completes the proof of the sufficiency of the condition.

To prove the necessity, let $R$ be an e.a.r., and $I$ be an ideal of $R$. Then $e(I)=e(x)=e(x R)$ for some $x \in R$. Since $R$ is an e.a.r., $I=r(e(I))=r(e(x R))=x R$, so that any commutative e.a.r. is a principal ideal ring.

In particular, $R$ is noetherian and so [2] $R$ is a subring of a ring $S$ which has descending chain condition on ideals, and hence specifically on annihilators. The latter chain condition is inherited by subrings, so $R$ has descending chain condition on annihilators. But every ideal of $R$ is an annihilator, so $R$ has descending chain condition on all ideals. Such a ring is well known to be decomposable into a direct sum of completely primary rings.

Suppose that $R$ is an e.a.r. and $R=S \oplus T$. Let $I$ be an ideal of $S$. Then $I$ is an ideal of $R$, so $I=e_{R}(a)$ for some $a \in R$. In the direct sum decomposition, let $a=b+c$. Then it is a triviality that $I=e_{S}(b)$, which shows that a direct summand of an e.a.r. is again an e.a.r. Hence all of the completely primary summands of $R$ are e.a.r.'s and consequently principal ideal rings, completing the proof of the theorem.

The theorem shows that in the commutative case, where a ring is both a l.e.a.r. and a r.e.a.r., if it has either condition, elemental annihilator rings have both chain conditions. The next theorem shows that this is also the case when $R$ is noncommutative, under the additional assumption that $R$ is semiprime (has no nonzero nilpotent ideals).

THEOREM II. Let $R$ be a semiprime ring. Then the following are equivalent:

(1) $R$ is both a r.e.a.r. and a l.e.a.r.

(2) $R$ is a l.e.a.r. and has ascending chain condition on right annihilators.

(3) $R$ is a direct sum of matrix rings over division rings.

Proof. That condition (3) implies both conditions (1) and (2) is clear.

To prove that (2) implies (3), let $R$ be a l.e.a.r. with ascending chain condition on right annihilators. Suppose that $L_{1} \supseteq L_{2} \supseteq \cdots$ is a descending chain of left ideals in $R$. For each $i, L_{i}=e\left(r\left(L_{i}\right)\right)$ since $R$ 
is a 1.e.a.r. and it is clear that $r\left(L_{1}\right) \subseteq r\left(L_{2}\right) \subseteq \cdots$. Hence there is an integer $k$ such that $r\left(L_{k}\right)=r\left(L_{n}\right)$ for all $n \geqq k$. Thus for $n \geqq k$, $L_{k}=e\left(r\left(L_{k}\right)\right)=e\left(r\left(L_{n}\right)\right)=L_{n}$ so that $R$ is a left Artin ring. Then $J$, the Jacobson radical of $R$, must be nilpotent and hence zero since $R$ is semiprime. The conclusion now follows from Wedderburn's theorem.

Finally, we prove that (1) implies (3). Let $R$ be both a l.e.a.r. and a r.e.a.r. Note that since $R$ is semiprime, it has no total left annihilators, and therefore if $x \in R, e(x)=e(x R)$. Let $I$ be a right ideal of $R$. Since $R$ is a r.e.a.r., $I=r(e(I))$. Also, since $R$ is a l.e.a.r., $e(I)=e(x)$ $=e(x R)$ for some $x \in R$. Then $I=r(e(I))=r(e(x R))=x R$, whence $R$ is a right principal ideal ring. But a semiprime right principal ideal ring is a finite direct sum of prime rings [3]. Each of these is again both a r.e.a.r. and a l.e.a.r. by an argument similar to that used in Theorem I, and so the argument above applies to show that they are principal right ideal rings also. Any prime right principal ideal ring [3] is a matrix ring $D_{n}$ over an Ore domain $D$. Let $L$ be a left ideal of $D$, then $L_{n}$, the $n$ by $n$ matrices over $L$, is a left ideal of $D_{n}$. Since $D_{n}$ is a l.e.a.r., there is an element $A=\left(a_{i j}\right)$ in $D_{n}$ such that $L_{n}=e(A)$. This means that $L=e_{D}(S)$, where $S$ is the set of $a_{i j}$. Thus every left ideal of $D$ is an annihilator, and in like manner every right ideal of $D$ is an annihilator. Since $D$ is an integral domain, this clearly forces $D$ to be a division ring, completing the proof of the theorem.

Note that the proof of the fact that (2) implies (3) uses only the fact that every left ideal of $R$ is a left annihilator, since this is sufficient for $L=e(r(L))$. This leads to the following.

COROLIARy. Let $R$ be semiprime with ascending chain condition on right annihilators. Then $R$ is a l.e.a.r. if and only if every left ideal of $R$ is the annihilator of some subset.

It would clearly be of interest to investigate the relation between the two classes of rings more closely, and to consider the consequences of removing the e.a.r. condition from one side in the statement of Theorem II.

\section{REFERENCES}

1. E. Snapper, Completely primary rings. IV, Ann. of Math. 55 (1952), 46-64.

2. L. Small, Studies on non-commutative noetherian rings, Ph.D. Thesis, Univ. of Chicago, Chicago, Ill., 1965.

3. C. Procesi, Sugli anelli principali ed un teorema di Goldie, Rend. Accad. Lincei 37 (1964), 804-807.

WASHINGTON UNIVERSITY 